\title{
Trans-sphenoidal Approach to the Supraclinoid Internal Carotid Artery for Endovascular Access in a Cadaver
}

\author{
Andrew Kelly Johnson, MD, MSE${ }^{1}$, Hunter Kegan Holt, BA², \\ Anthony Joseph Serici, BS ${ }^{3}$, Roham Moftakhar, MD'
}

Purpose: Sometimes, intracranial pathology in the distal vasculature cannot be accessed by standard endovascular techniques because of occlusion or insurmountable tortuosity of theinternal carotid artery (ICA). A trans-sphenoidal surgical approach can follow a similar trajectory to the course of the supraclinoid ICA. This study evaluates the feasibility of a trans-sphenoidal approach to the supraclinoid ICA for endovascular access.

Materials and Methods: In a fresh cadaver head, the sphenoid sinus was dissected through a trans-sphenoidal route. Bone over the carotid prominence was removed to expose the ICA. The artery was catheterized using the Seldinger technique, and three-dimensional digital subtraction angiography was performed to evaluate the procedure.

Results: The catheter was successfully inserted into the supraclinoid ICA via the trans-sphenoidal route. Three-dimensional radiographic reconstruction confirmed placement of the catheter and the trajectory of the sheath into the supraclinoid ICA.

Conclusion: While the trans-sphenoidal route has innumerable disadvantages over the standard endovascular access techniques, this route could be considered when other treatment options are too risky or impractical.

Key Words : Trans-sphenoid; Endovascular; Internal carotid artery

${ }^{1}$ Department of Neurological Surgery and ${ }^{3}$ Department of Anatomy, Rush University Medical Center, Chicago, IL, USA, ²Rush Medical College, Chicago, IL, USA

Received January 28, 2013; accepted after revision February 19, 2013.

Correspondence to: Andrew K. Johnson, MD, Department of Neurological Surgery, Rush University Medical Center, Chicago, IL, 1725 W. Harrison St.Suite 855, Chicago, IL 60612, USA.

Tel. +1.312.942.6644 Fax. +1.312.563.3358

E-mail: andrew_johnson@rush.edu

This is an Open Access article distributed under the terms of the Creative Commons Attribution Non-Commercial License (http://creativecommons.org/licenses/by-nc/3.0) which permits unrestricted non-commercial use, distribution, and reproduction in any medium, provided the original work is properly cited.
The scope of endovascular contributions to the management of intracranial pathology continues to expand. Achieving stable endovascular access is rarely a concern due to intracranial anastomoses and innovation of techniques and devices. Occasionally, however, an intracranial artery may be inaccessible due to proximal occlusion or tortuosity, which would prevent endovascular management. Direct cervical puncture can overcome the occlusion of the proximal internal or the common carotid arteries (CCA) $[1,2]$, but it is possible depending on the point of arterial obstruction and the available length of artery to be punctured. 
Distal access can be obtained through craniotomy, but puncture of intracranial vessels and endovascular navigation beyond the puncture site has not been described to our knowledge.

Trans-sphenoidal surgery generally requires avoidance of the anterior bend of the carotid siphon. During pituitary surgery, the sella is identified between the usual bony prominences overlying this segment of the ICA. Anatomical studies have shown that the superior-most aspect of the carotid prominence into the sphenoid sinus, the presellar bony segment, can be visualized in $98 \%$ of specimens [3]. Behind this segment, the carotid artery often travels between the lower and the upper dural rings before entering the subarachnoid space. The relationship of the transsphenoidal approach and the ICA provides two key
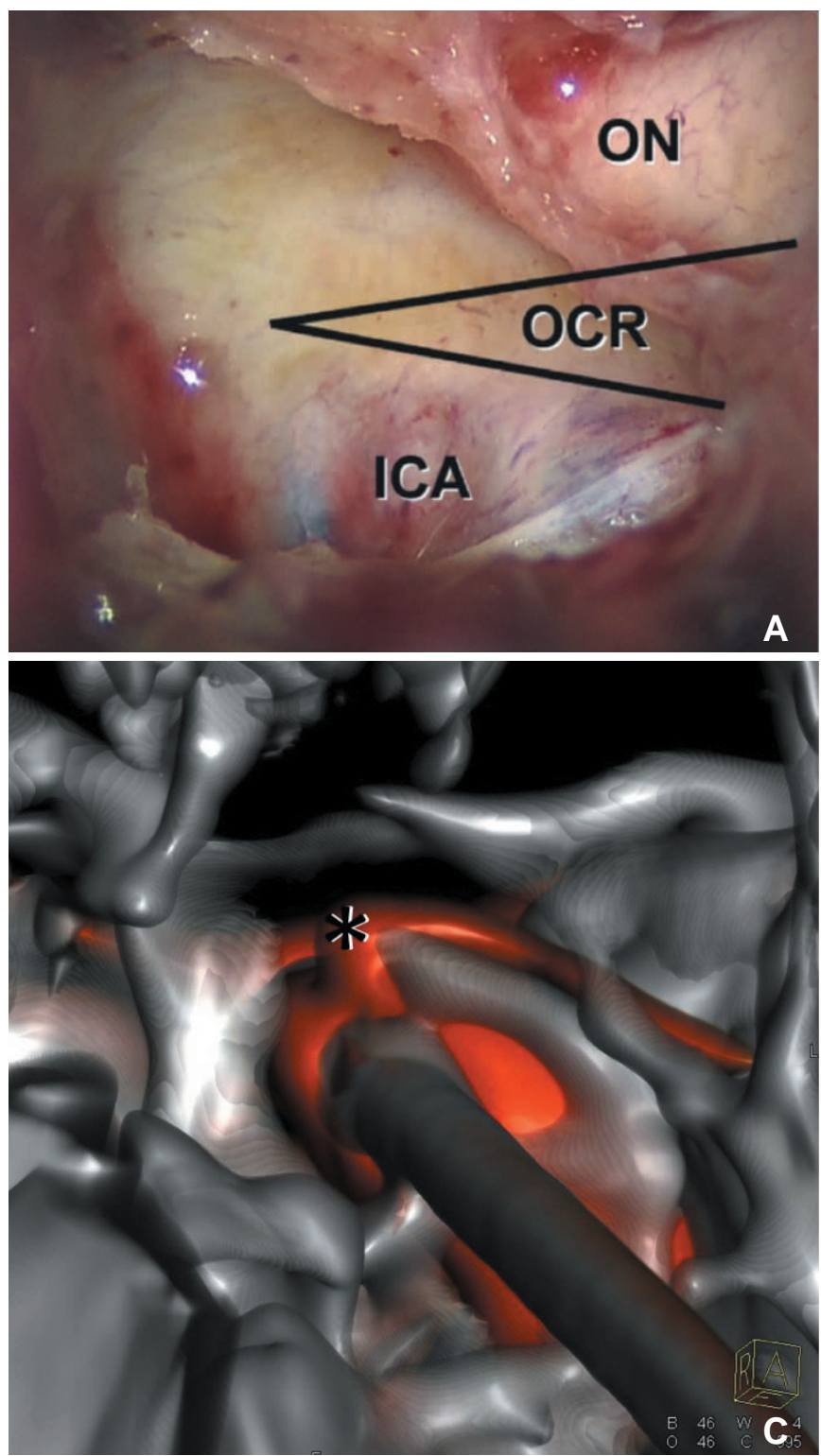

features aiding catheterization of the supraclinoid ICA. First, the anterior bend of the ICA allows puncture of the artery at an obtuse angle under direct visualization. Second, the supraclinoid ICA travels posteriorly and superolaterally in a trajectory quite similar to that between the contralateral naris and this segment of the ICA. In this study, we catheterized the supraclinoid ICA of a human cadaver through a trans-sphenoidal approach and assessed the viability of the technique radiographically.

\section{MATERIALS AND METHODS}

The sphenoid sinus was accessed via trans-nasal, microscope-assisted dissection of a fresh cadaver. The sphenoid sinus was opened at both ostia and widened

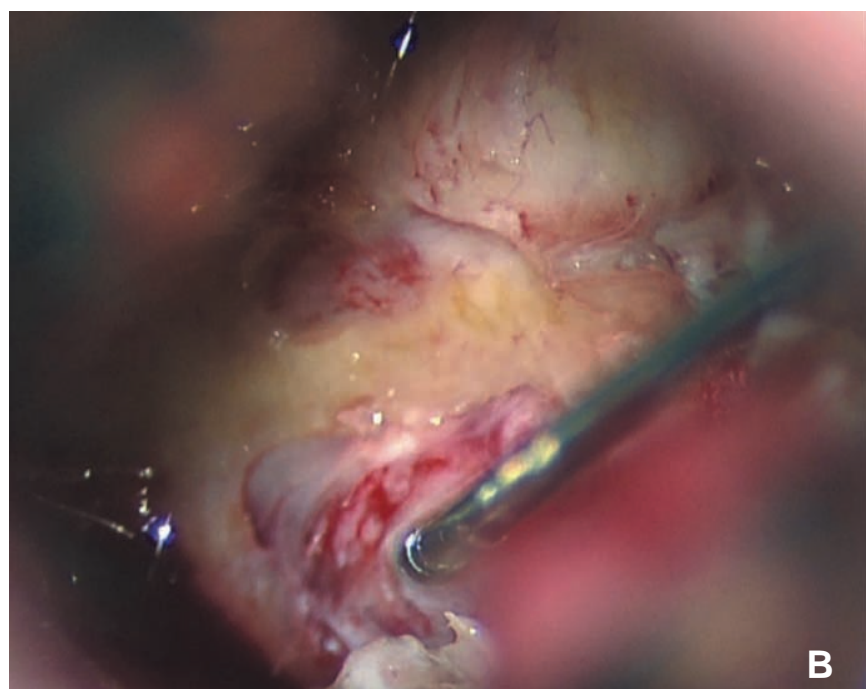

Fig. 1. (A) Microscopic view through the right naris into the left superolateral sphenoid sinus demonstrates the lateral opticocarotid recess (OCR) and bony prominences over the optic nerve $(\mathrm{ON})$ and internal carotid artery (ICA), (B) wire access from the left naris into the left ICAprior to the cannulation with the access catheter, (C) dual volume three-dimensional reconstruction image of skull base and vessels shows the entrance of the catheter into the left supraclinoid ICA and the origin of ophthalmic artery $\left({ }^{*}\right)$, which was not visualized during dissection. 

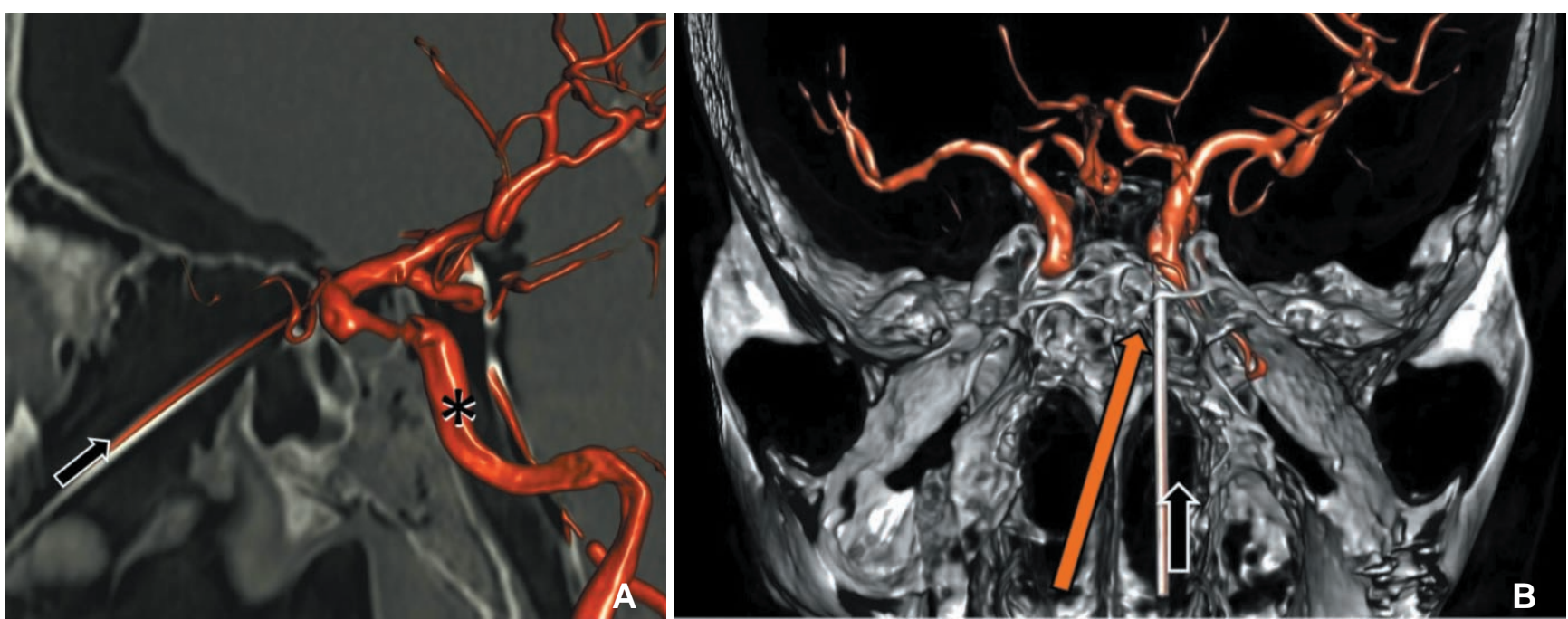

Fig. 2. Dual volume reconstruction images of skull base and vessels after contrast injection into the trans-sphenoidal catheter (A) sagittal embedded multiplanar reconstruction shows the trajectory of the catheter (black arrow) entering the left ICA ( $\left.{ }^{*}\right)$ in its supraclinoid segment from the ipsilateral naris. (B) Access through the contralateral naris might improve axial trajectory (orange arrow).

anterolaterally. The microscope viewing angle was directed through the right naris toward the left lateral opticocarotid recess (Fig. 1A). The bony presellar carotid prominence overlying the cavernous carotid artery was drilled to expose the artery (Fig. 1B). The Seldinger technique was used to gain access to the left supraclinoid ICA with a needle that punctured the visualized ICA from the left naris (Fig. 1C). A 4 Fr, 11 $\mathrm{cm}$ Avanti introducer sheath with dilator (Cordis, Miami Lakes, FL, USA) was advanced along a wire, and the wire and dilator were removed. The sheath, the tip of which was previously marked with ink, was inserted to a depth of 8-10 mm. After placement, the sheath was sutured to the cartilaginous nasal septum.

To confirm adequate placement of the sheath, the cadaver was then examined with DynaCT (Siemens, Erlangen, Germany). Eight second three-dimensional digital subtraction angiography with injection of $24 \mathrm{~mL}$ of $50 \%$ Isovue- 250 through the sheath was performed. Vascular access, relationship between ICA and sheath trajectory were then analyzed using the Siemens Leonardo workstation with InSpace three-dimensional reconstruction.

\section{RESULTS}

Access to the supraclinoid ICA was successfully achieved with the trans-sphenoidal approach. Dual volume reconstruction showed the sheath, skull base, and arteries with no apparent contrast extravasation into the nasal sinuses or subarachnoid space.

Dual volume images clipped parallel to the sheath demonstrated excellent alignment in the sagittal plane (Fig. 2A). An oblique axial slab showed adequate alignment (Fig. 2B). Reconstruction also revealed the origin of the ophthalmic artery, which was not seen during dissection and vessel catheterization.

\section{DISCUSSION}

The trans-sphenoidal route provided viable access to the left supraclinoid ICA in this study. We inserted an introducer sheath through the left naris during the direct microscope visualization through the contralateral right naris. It would be better to align the sheath trajectory to the left supraclinoid ICA through the right naris; this contralateral approach may be easier using endoscopic visualization through either naris. In comparison to transfemoral access, placement of a sheath directly into the supraclinoid ICA has advantages of maximum device stability for endovascular intervention and minimum arterial tortuosity to optimize responsiveness of microwires and other endovascular devices. A 4 Fr sheath would both provide greater access than the largest distal access catheters because there is no need for the guiding catheters with larger diameter and minimize the size of the puncture. However, the technique could be expanded to larger catheters if needed.

Very rarely, this approach may be the only option. Endovascular treatment of a recurrent ICA aneurysm previously treated with incomplete trapping could be accomplished through this route. An acute ischemic stroke caused by a middle cerebral artery (MCA) 


\section{Andrew Kelly Johnson, et al.}

thromboembolus often requires distal access with large catheters for thrombectomy. If a stenotic, diseased cervical carotid artery and small communicating arteries prevent access through standard methods, the trans-sphenoidal route may be considered for the MCA access. Any situation where the carotid siphon is too tortuous to navigate with a larger device, the transsphenoidal route would allow direct, minimally convoluted access to the supraclinoid ICA and proximal MCA. Finally, during skull base surgery for a tumor that has invaded the internal carotid artery, injury to the petrous or cavernous segments may be difficult to manage. These procedures often provide access to the sphenoid sinus, and puncture of the clinoidal ICA may be a fast maneuver to provide distal arterial control, thus allowing the surgeon to attempt repair of the internal carotid artery instead of sacrificing it.

Disadvantages to trans-sphenoidal endovascular access make it an option in only rare circumstances. First, neurointerventionalists not trained in skull base surgery would require an access surgeon, and there is morbidity and discomfort associated with any transsphenoidal surgery. Second, endonasal surgery is not sterile, and compared to transfemoral access, there is a theoretical risk of infection seeding to the carotid arterial distribution, cavernous sinus, or meninges. However, endonasal surgery has not been shown to have an increased infection risk compared to open surgeries including craniotomy [4], though this $1.8 \%$ infection rate is certainly significant compared to the negligible rate of standard transfemoral endovascular access.

Specific anatomical concerns include the ophthalmic artery origin and location of the dural rings. The ophthalmic artery has a cavernous origin in $8 \%$ of cases [5], and puncture or avulsion could lead to ipsilateral blindness and challenging bleeding. The length of the extracavernous-prearachnoid or clinoid ICA segment between the dural rings, the ideal puncture site, is approximately $3.1 \mathrm{~mm}$ in length but varies from $0.5-$ $6.5 \mathrm{~mm}$ [6]. Puncture above the upper dural ring superior to the clinoid segment of the ICA could produce a cerebrospinal fluid leak or subarachnoid hemorrhage, and puncture below the inferior dural ring could produce a carotidcavernous fistula. But consistent localization of the segment between the dural rings with preoperative imaging may not be realistic.

The biggest concern with trans-sphenoidal endovascular access is closure of the puncture site, assessment of which was not possible in our cadaver study. Beyond initial closure, more insidious complications of pseudoaneurysm or carotid-cavernous fistula formation increase the overall morbidity risk. The technique does offer two advantages over trans-femoral access related to closure. First, the actual puncture is smaller in diameter and performed under direct visualization; second, direct endoscopic or microscopic visualization of the puncture site can help focal manual compression with a thrombotic agent. An intravascular balloonextravascular thrombostatic plug mechanism similar to that employed by the Mynx closure device (AccessClosure, Mountain View, CA, USA) would potentially be effective for arteriotomy closure under direct visualization. Until a punctured internal carotid artery can be safely and consistently sealed after a trans-sphenoidal endovascular procedure, use of this route for endovascular access should be limited to extreme circumstances.

One additional complication could be unexpected loss of access due to sheath pullout. Access depth of approximately one centimeter and a suture in the naris were adequate for securing the sheath during manipulation in the current study. However, the neurointerventionalists must be aware that an inadvertent move could leave a gushing hole in the artery if the sheath comes out.

There were several limitations to the current study. Endoscopy rather than surgical microscopy would have facilitated operative access to the lateral optico-carotid recess. Three dimensional image guidance could assist in optimizing both entry point and trajectory of catheter placement. These tools would help any attempt of this procedure. A single cadaver was investigated, and while the trajectory and landmarks were excellent, the study does not account for anatomical variation. The magnitude of bleeding issues from both the cavernous sinus and carotid artery could not be appreciated.

In conclusion, trans-sphenoidal approach to the supraclinoid ICA is unlikely to become a common endovascular practice, but it may provide the only probable access for a patient with difficult proximal anatomy when alternative options are too risky or impractical.

\section{Acknowledgment}

Special thanks to all of those who assisted in the execution of this project, specifically Arthur Maydell, MD and Joel Delacruz. This work was performed in the Human Anatomy Laboratory of the Rush University Medical Center, James M. Williams, PhD, Director. 
Trans-sphenoidal access to ICA

\section{References}

1. Halbach VV, Higashida RT, Hieshima GB, Hardin CW. Direct puncture of the proximally occluded internal carotid artery for treatment of carotid cavernous fistulas. AJNR Am J Neuroradiol 1989;10:151-154

2. Tsai YH, Weng HH, Chen YL, Wu YM, Wong HF. Treatment of recurrent carotid cavernous fistula by direct puncture of a previously trapped internal carotid artery. J Vasc Interv Radiol 2010;21:738-740

3. Rhoton AL Jr. The sellar region. Neurosurgery 2000;51:S335-374
4. Kono Y, Prevedello DM, Snyderman CH, Gardner PA, Kassam $\mathrm{AB}$, Carrau RL, et al. One thousand endoscopic skull base surgical procedures demystifying the infection potential: incidence and description of postoperative meningitis and brain abscesses. Infect Control Hosp Epidemiol 2011;32:77-83

5. Rhoton AL Jr. The supratentorial arteries. Neurosurgery 2002;51: S53-120

6. Alencastro LC. The anterior loop of the carotid siphon. Skull Base Surg 1991;1:73-77 